\title{
Ultra-short-term Radiation Prediction based on FY-4 Satellite Cloud Images and Artificial Neural Network
}

\author{
Dongyu $\mathrm{Jia}^{1^{*}}$, Xiaoying $\mathrm{Nie}^{1}$, Fuyuan $\mathrm{Gao}^{1}$, Qingfeng $\mathrm{Li}^{1}$ \\ ${ }^{1}$ College of Geography and Environmental engineering, Lanzhou City University, Lanzhou 730070, China
}

\begin{abstract}
Surface solar radiation is affected by many random mutation factors, which makes the ultra-shortterm prediction face great challenges. In this paper, the surface radiation observation station in the northwest (Dunhuang) desert area with broad PV prospects is selected as the research object. The input parameters of the test sample are: cloud forecast value, reflectivity and brightness temperature value of a satellite cloud image closest to the forecast time. The MATLAB software is used to model the prediction program and to predict the surface solar radiation in the next 10 minutes. A combined algorithm of satellite cloud images and neural network is applied to predict surface solar radiation for the next 10 minutes and is compared with the measured surface solar radiation. The model is a lightweight calculation model, it satisfies the calculation precision of engineering requirements. The results show that the diurnal variation trend of measured and predicted radiation values is basically the same. Among them, the prediction accuracy of the model for cloudy days is higher, while for snowy days with more abrupt changes, the prediction error of abrupt points is larger. The model can provide reference for ultra-short-term prediction of surface radiation.
\end{abstract}

\section{Introduction}

Due to the limited reserves of petroleum, coal and other resources, the development of new energy is an inevitable trend. Photovoltaic power generation will become an important part of the future energy structure. When photovoltaic power generation accounts for more than $15 \%$ of the power system, the fluctuation of photovoltaic power generation may cause the power system to be paralyzed ${ }^{[1]}$. Therefore, the power forecast of photovoltaic power station is very important to the safety and stability of the whole power grid. The fluctuation of solar radiation is the main factor affecting photovoltaic power generation.

At present, many scholars have analyzed and studied the prediction methods of surface solar radiation ${ }^{[1-8]}$. Chen et al. ${ }^{[2]}$ established the error prediction equation through the error stepwise approximation method and established an improved solar radiation prediction method. Ding et al. [3] used the full-sky imager and established the cloud radiation attenuation model to predict the total radiation received from the ground. Chen et al. ${ }^{[1]}$ used ground-based cloud map and neural network to model surface radiation prediction. Steven et al. ${ }^{[4]}$ established a short-term (0-3h) surface solar radiation prediction method by using geostationary satellite observation and numerical weather forecast. Rosiek et al. ${ }^{[5]}$ used satellite observation and artificial neural network to establish online 3-hour power output forecast of photovoltaic building integration system. Ayet et al. ${ }^{[6]}$ studied the approach (6h) forecast of solar irradiance by using static satellite images and numerical simulation. At present, the difficulty in the study of ultra-short term prediction of surface solar radiation lies in the movement and dissipation of clouds. At present, the observation of clouds is mainly based on ground cloud map and satellite cloud map. The time and space resolution of ground cloud map is relatively high, but the observation range is limited. The successful launch of the current Fengyun-4A satellite enables China's quantitative remote sensing satellite in geostationary orbit to reach the world's advanced level. Fengyun-4A satellite has a high temporal and spatial resolution and can better predict cloud movement. Due to the late start of the domestic photovoltaic industry, the ultra-short term radiation forecast of photovoltaic power generation is still in the exploratory stage.

Taking Dunhuang observation station in northwest China as the research object, the observation data of Fengyun-4 geostationary satellite is used to determine cloud cover and forecast cloud movement. Combined with artificial neural network, the total solar radiation received on the surface in the future $10 \mathrm{~min}$ has been predicted. Northwest China has abundant solar energy resources and broad prospects for developing photovoltaic power stations. For the desert area of northwest China where there are few meteorological stations, the satellite cloud map data is very precious. This study can provide reference for ultra-short term radiation prediction of photovoltaic power generation. 


\section{Data and Methods}

\subsection{Data}

Dunhuang Shuangpier observation point, latitude: $40^{\circ} 09$ '29.1 ", longitude: $94^{\circ} 30^{\prime} 58.7$ ", altitude: $1148 \mathrm{~m}$, the type

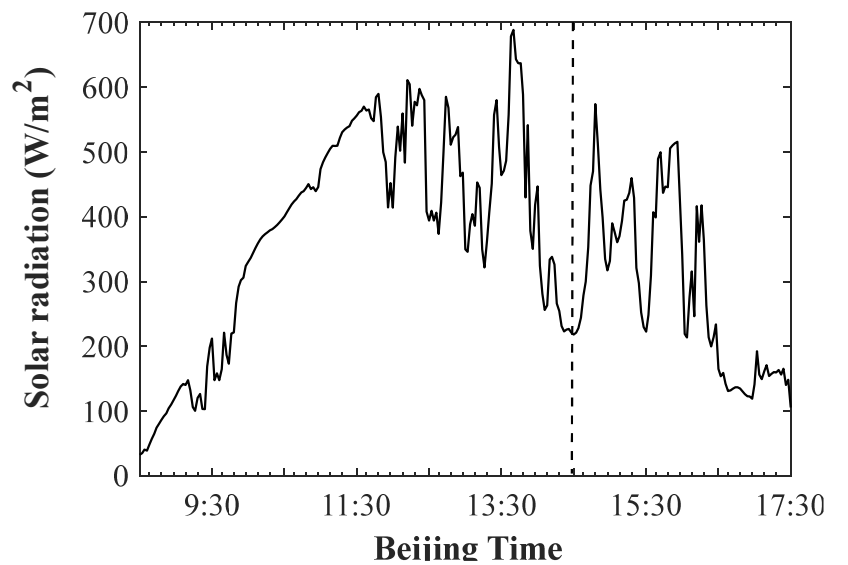

of underlying surface is sand and stone Gobi. The observed data are the four components of radiation. The sensor is the Kipp\&Zonen CNR4 net radiation sensor, and the acquisition frequency is $2 \mathrm{~min} /$ time. The observation time is from November to December 2018.

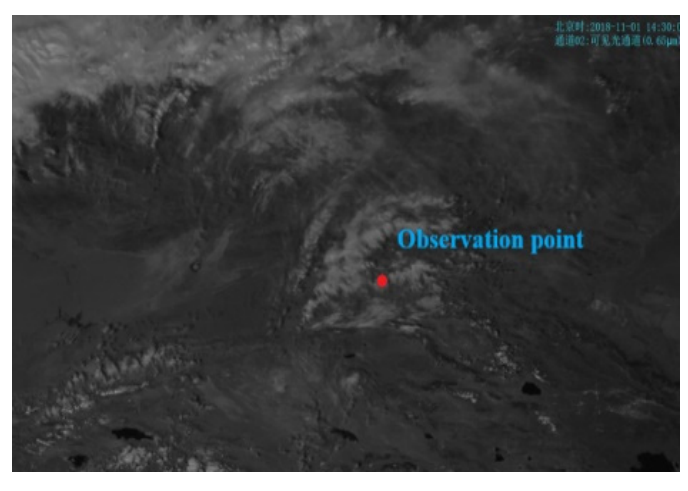

Figure 1. Diurnal variation of surface radiation observation stations on November 1,2018 and satellite cloud data at $14: 30$ of the day

\subsection{Methods}

\subsubsection{Calculation of cloud cover}

In the FY-4A satellite imager 500ML1 image, four grid points are selected as the center and combined with cloud detection data, the ratio of the number of grid points inverted to the total number of selected grid points is the cloud cover data of the place ${ }^{[9]}$.

$$
C=\frac{N_{\text {cloud }}}{N_{\text {cloud }}+N_{\text {sky }}}
$$

\subsubsection{Cloud movement vector calculation}

Previous studies have shown that this method has a high application value in the field of fluid mechanics ${ }^{[10]}$. In this paper, particle image velocity measurement software 'MPIV' based on MATLAB developed by Mori and Chang ${ }^{[11]}$ has also been used to calculate the velocity field of cloud cluster. As shown in Fig. 2, the satellite cloud images at 14:34 and 14:38 on November 1, 2018 at the observation point are used to calculate the cloud movement vector which is more in line with the actual situation through the calculation of velocity measurement software and the improvement measures of cloud filtering, smoothing and quality control. The velocity vectors $\mathrm{u}=2.70$ and $\mathrm{V}=1.20$ (pixels $/ \mathrm{min}$ ) of cloud motion are obtained.

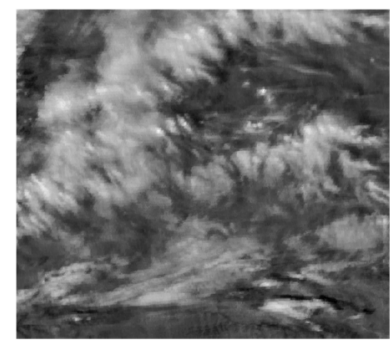

a. 14:34 Beijing Time

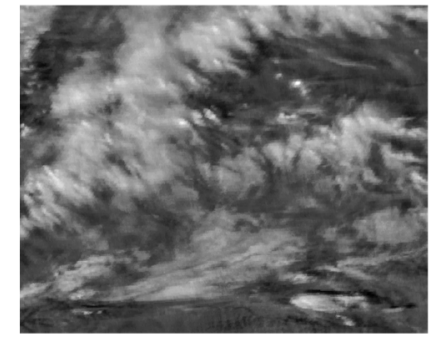

b. 14:38 Beijing Time

Figure 2. Satellite Cloud Images on November 1, 2018

\section{Analysis of forecast results}

We used the prediction process in Fig. 4 to predict the surface solar radiation value of Dunhuang Shuangpier

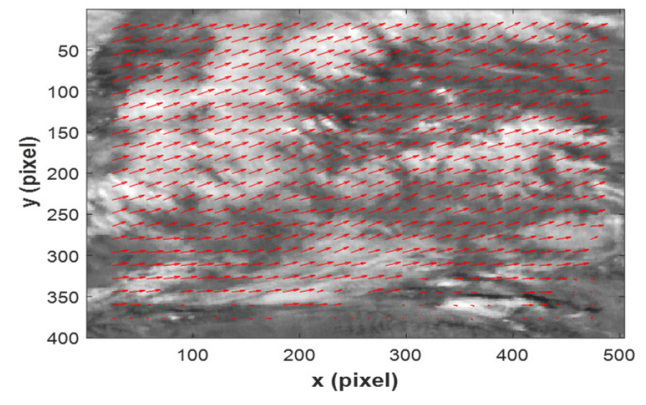

Figure 3. Velocity Vector Corresponding to MPIV

observation station in the future $10 \mathrm{~min}$. Because this paper focuses on cloud information, the forecast results with cloud sky are compared with the measured values. Fig. 4 shows the forecast results, among which Fig. 4a shows cloudy days and Fig. $4 \mathrm{~b}$ shows light snow days. It can be seen from the figure that the model can well predict the 
basic trend of solar radiation on the surface. For cloudy days, the prediction accuracy is relatively high, the correlation coefficient between the measured radiation value and the predicted radiation value reaches 0.93 , the root mean square error (RMSE) is $53.3 \mathrm{~W} / \mathrm{m} 2$, the average absolute error (MAE) is $43.3 \mathrm{~W} / \mathrm{m} 2$, and the average relative error (MRE) is $1.1 \%$. The value with a large error occurs from 14:00 to $14: 30$, mainly because there are only two satellite cloud images in the period from 13:38 to 14:30, with a large time interval, making the error larger.
For the forecast of light snow days, the prediction accuracy is larger than that of cloudy days. The RMS error reaches $89.8 \mathrm{~W} / \mathrm{m} 2$, the average absolute error is 69.5 $\mathrm{W} / \mathrm{m} 2$, and the average relative error is $9.9 \%$. It can be seen from Fig. 4b that the forecast error of the abrupt transition point of snow-day radiation is large, and the forecast value is relatively discrete from the measured value. However, compared with the forecast results without cloud map information ${ }^{[12]}$, this model still significantly improves the forecast results.

(a)
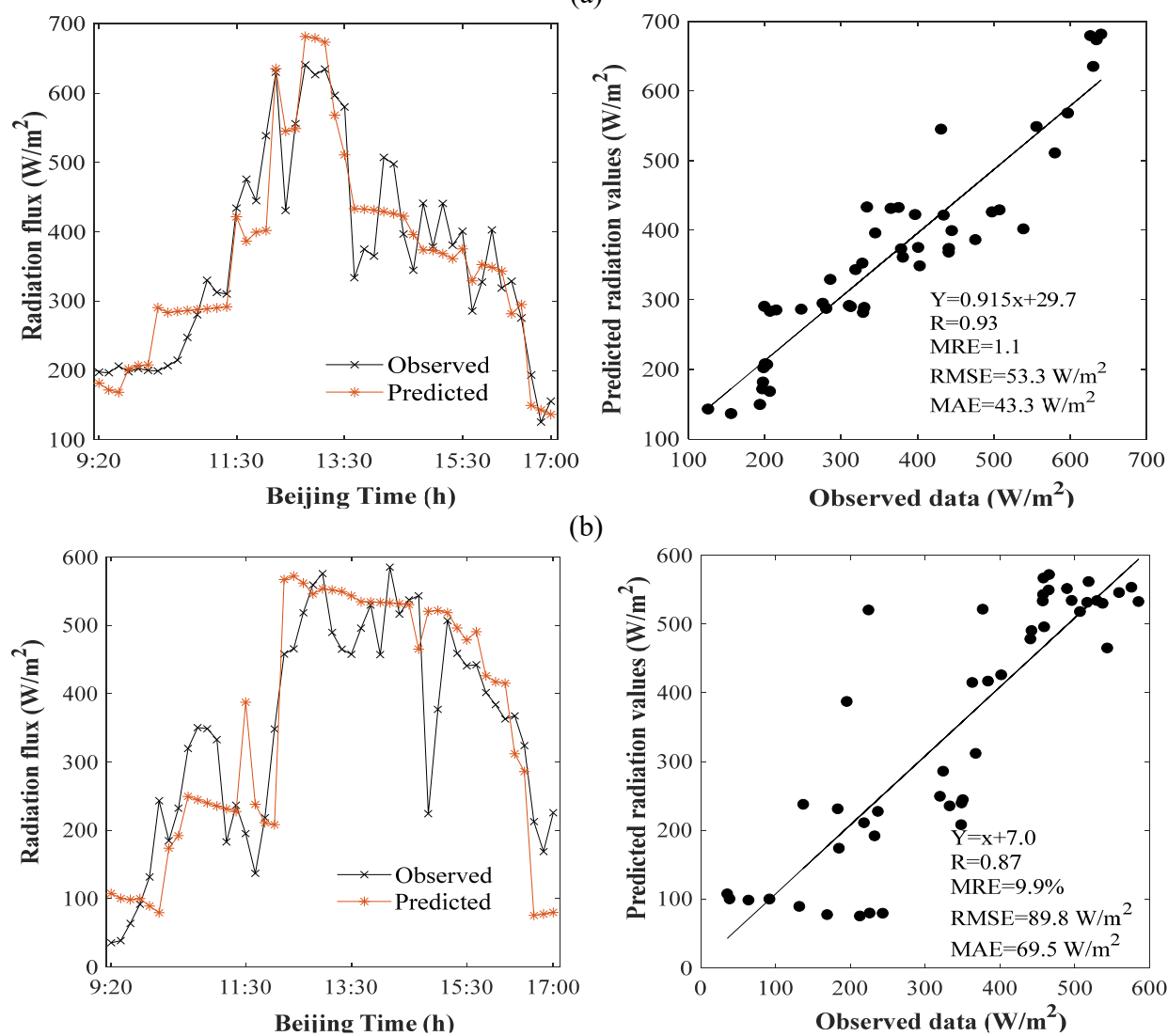

Figure 4. The forecasting values of surface solar radiation by satellite cloud images and neural network (a) Forecast value of surface solar radiation on November 04, 2018 (cloudy) (b) Surface solar radiation forecast value for November 17, 2018 (light snow)

\section{Conclusion and discussion}

For the desert area of northwest China where there are few meteorological stations, satellite data is very precious. In this paper, the numerical value of solar radiation received from the ultra-short term surface is predicted by using the algorithm of cloud map of Fengyun-4 satellite and artificial neural network. On the premise of ensuring high accuracy, the number of variables and the amount of data selected are small, which is a lightweight prediction model. It can be seen from the forecast results that the model can well predict the basic trend of surface solar radiation. For cloudy days, the prediction accuracy is relatively high, while for snow days with many abrupt changes, the prediction error of abrupt changes is relatively large, so the accuracy needs to be further improved.
Cloud movement, generation and elimination is an extremely complex process affected by many factors. Although the cloud image of The Fengyun- 4 satellite has a high spatial and temporal resolution, there is also a time interval between two cloud images. Although the observation range of the ground cloud map is limited, the spatial and temporal resolution is higher. Therefore, based on the satellite image and the ground cloud image, radiation prediction will be the next step.

\section{Acknowledgments}

This work is financially supported by the Doctoral Research Fund of Lanzhou City University (Grant LZCUBS2019-13) and the Opening Fund of the Key Laboratory of Land Surface Process and Climate Change in Cold and Arid Regions, CAS (Grant LPCC2018006). 


\section{References:}

1. Z B Chen, J Ding, H Zhou, et al., Proceedings of the CSEE, 35, 561-7 (2015)

2. Z H Chen, P J Sun and R Zhang, Acta Energiae Solaris Sinica, 36, 2377-83 (2015)

3. Y Y Ding, J Ding, H Zhou, et al., Proceedings of the CSEE, 34, 50-6 (2014)

4. Miller, D Steven, Rogers, et al., Solar Energy, 168, 102-17 (2018)

5. S Rosiek, J Alonso-Montesinos and F J Batlles, Electrical Power and Energy Systems, 99, 261-72 (2018)

6. A Ayet and P Tandeo, Solar Energy, 164 301-15 (2018)

7. X Q Gao, J X Jun, N B Wang, et al., Acta Energiae Solaris Sinica, 1, 95-100 (2018)

8. Y Cui, C Cheng and Z H Chen, Water Resources and Power, 36, 201-4 (2018)

9. G Caili, Beijing, China Meteorological Press, 494 (2004)

10. Y Yang, X D Ruan and H Y Yang, Mechanical \& Electrical Engineering Magazine, 22, 1-4 (2005)

11. N Mori and KA Chang, http://www.oceanwave.jp/softwares/mpiv (2003)

12. L W Yang, X Q Gao, J X Jiang, et al., Acta Energiae Solaris Sinica, 41, 152-7 (2020) 